\title{
Chor-Rivest Knapsack Cryptosystem in a Post-quantum World
}

\author{
Raúl Durán Díaz, Luis Hernández-Álvarez, Luis Hernández Encinas, \\ and Araceli Queiruga-Dios
}

\section{Introduction}

It is known that the quantum algorithms proposed by Shor [1] will break the main asymmetric cryptosystems used today, if a quantum computer with sufficient computing capacity is developed (it is estimated that with more than $10^{7}$ qubits). Indeed, the mathematical problems on which their security is based (integer factorization and discrete logarithms) could be solved in just a few hours. In fact, if a current PC needs $\mathcal{O}\left(2^{\sqrt[3]{\log n}}\right)$ bit operations to break an algorithm, a quantum computer using Shor algorithm could reduce such number of bit operations to $\mathcal{O}\left(\log ^{3} n\right)$ while requiring a memory storage of $\mathcal{O}(\log n)$ bits.

As to symmetric cryptography, Grover [2] and Simon [3] algorithms could reduce the computing time required to break the main symmetric cryptosystems to the square root of the current time. That is, if a quantum computer with the sufficient computing capacity is developed, the security of current symmetric cryptosystems would be equivalent to that of the same cryptosystems with half-length keys.

Due to these quantum threats to the security of the information when it is protected with the current cryptosystems, the National Institute of Standards and

\footnotetext{
R. Durán Díaz

Departamento de Automática, Universidad de Alcalá, Alcalá de Henares, Spain e-mail: raul.duran@uah.es
}

L. Hernández-Álvarez · L. Hernández Encinas $(\bowtie)$

Instituto de Tecnologías Físicas y de la Información (ITEFI), Consejo Superior de Investigaciones Científicas (CSIC), Madrid, Spain

e-mail: luis.hdez.alvarez@iec.csic.es; luis@iec.csic.es

\footnotetext{
A. Queiruga-Dios

Departamento de Matemática Aplicada, Universidad de Salamanca, Salamanca, Spain

e-mail: queirugadios@usal.es
} 
Technology (NIST) has launched an International Call to select new quantumresistant cryptographic algorithms [4] for asymmetric encryption, digital signatures, and key encapsulated mechanisms. In January 2019, NIST published the list of the algorithms that had been promoted to the second round [5]. The security of such algorithms is based on specific mathematical problems founded in lattices, error correction codes, quadratic multivariate polynomials, hash functions, and elliptic curve isogenies. The objective of NIST is to select new cryptographic systems invulnerable to quantum computers (or quantum resistant), no matter how much computing power they have.

On the other hand, traditionally, cryptosystems based on knapsack problems have had bad press because most of them have been completely broken. In this sense, it is important to note that Chor-Rivest cryptosystem $[6,7]$ was one of the systems only broken for the original parameters proposed [8]; whereas it has been proved that it is possible to select safer parameters $[9,10]$ which could permit to implement such cryptosystem in a secure way.

In this paper, we present the state of art of some of the mathematical problems proposed to define quantum-resistant asymmetric cryptosystems [11] and moreover, we want to focus on another problem, not considered so far, that could offer alternatives of interest for post-quantum security. This is a knapsack-type problem, which uses the arithmetic of finite fields and needs to compute discrete logarithms in order to determine the keys of the system. The interesting point is that the security of such cryptosystem depends on the knapsack problem but not on the discrete logarithm problem (DLP). In fact, if the DLP becomes tractable, then the ChorRivest cryptosystem is easier to implement, but not easier to break.

The rest of this paper is organized as follows: in Sect. 2 we present the most important mathematical foundations upon which the new cryptographic proposals are based. Section 3 shows an alternative cryptographic primitive, not considered in the NIST call, that could be added to the list of quantum-resistant algorithms. Finally, Sect. 4 presents a discussion about the possible benefits that such proposal could offer to the quantum scenario and some future works.

\section{Post-quantum Proposals}

We focus our attention over asymmetric cryptography since as we have mentioned before, it seems to be the most affected by quantum algorithms. The most important proposals, which will be dealt with in turn, are: (1) hash-based, (2) code-based, (3) lattice-based, (4) multivariate-quadratic-equations, and (5) elliptic-curve-isogenybased. 


\subsection{Hash-Based Cryptography}

This proposal stems from an old one-time signature scheme due to Lamport and Diffie [12] and provides a quantum-resistant signature scheme. The key ingredient is a hash function $H$, which is required to be simply pre-image resistant (this means that an adversary provided with $x, y$, with $y=H(x)$, is not able to determine $x^{\prime} \neq x$ such that $\left.y=H\left(x^{\prime}\right)\right)$. To fix ideas, let us assume that $H$ outputs $n$-bit hashes for any input message, i.e.,

$$
H:\{0,1\}^{*} \rightarrow\{0,1\}^{n}
$$

The first step is for the user to generate her public/private key pair. In order to do so, she selects uniformly at random $2 n n$-bit strings, which make up the secret key:

$$
S K=\left(s k_{n-1}[0], s k_{n-1}[1], \ldots, s k_{0}[0], s k_{0}[1]\right)
$$

and publishes the corresponding public key, namely

$$
P K=\left(p k_{n-1}[0], p k_{n-1}[1], \ldots, p k_{0}[0], p k_{0}[1]\right)
$$

where $p k_{i}[j]=H\left(s k_{i}[j]\right)$, for $0 \leq i \leq n-1, j=0,1$.

Given a message $M \in\{0,1\}^{*}$ to be signed, the user first computes $h=H(M)$, which is an $n$-bit length string, so that $h=\left(h_{n-1}, \ldots, h_{0}\right)$. The signature process yields the signature $\sigma$ for $M$ by setting

$$
\sigma=\left(\sigma_{n-1}, \ldots, \sigma_{0}\right)=\left(s k_{n-1}\left[h_{n-1}\right], \ldots, s k_{0}\left[h_{0}\right]\right)
$$

The verification of the pair $(M, \sigma)$ is accomplished as follows: the verifier computes $h=H(M)$, and then sets $h=\left(h_{n-1}, \ldots, h_{0}\right)$. The signature is deemed correct if

$$
H\left(\sigma_{i}\right)=p k_{i}\left[h_{i}\right], 0 \leq i \leq n-1 .
$$

Several remarks are in order here. First, it is clear that the public/private key pair can be used safely only once, which accounts for the term "one-time signature." Both private and public keys have $2 n^{2}$ bits, and the signature needs $n^{2}$ bits. For example, if one chooses SHA-256 as a hash primitive, then $n=256$, so the signature has a length of $n^{2}=65,536$ bits, and the key pair $2 n^{2}=131,072$ bits.

However, the key point is that the signature scheme just described remains safe as long as the underlying hash functions remain hard to invert. And the good piece of news is that this last statement is true even in the presence of quantum algorithms (for example, Grover's). Public key cryptography is saved at least for the time being. 


\subsection{Merkle Trees}

Merkle trees are a device intended to open the possibility of signing several messages and builds upon the one-time signature scheme above.

The user chooses a positive integer $\ell$, and generates $2^{\ell}$ key pairs $\left(X_{i}, Y_{i}\right), 0 \leq$ $i \leq 2^{\ell}-1$, following Lamport's one-time signature scheme. Equipped with these $\ell$ key pairs, a signer uses them up to exhaustion as long as she commits the public keys to some kind of "global public key." The latter can be accomplished by means of the so-called Merkle trees, which are binary trees such as the one shown in Fig. 1.

Let us denote the nodes as $n_{h}[k]$, where $h$ is the "floor number" and $k$ is the ordering inside a floor, from left to right, so that $0 \leq k \leq 2^{\ell-h}-1$ for floor number $h$. Merkle tree will be filled in as follows:

$$
\begin{aligned}
& n_{0}[k]=H\left(Y_{k}\right), 0 \leq k \leq 2^{\ell}-1, \\
& n_{h}[k]=H\left(n_{h-1}[2 k] \| n_{h-1}[2 k+1]\right),
\end{aligned}
$$

for $1 \leq h \leq \ell, 0 \leq k \leq 2^{\ell-h}-1$, where $\|$ is the "concatenation" operator. Once this operation is complete, the user publishes the root node value, $n_{\ell}[0]$, as the "global public key" and serves as a commitment of the whole set $Y_{i}$ of public keys.

To sign messages, the user takes the secret keys $X$ one at a time, until exhaustion. Assuming it is the time for the secret key $X_{s}$ to be used, the user signs a message using Lamport's one-time scheme with the pair $\left(X_{s}, Y_{s}\right)$, yielding the signature, say, $\sigma_{s}$. The user publishes this one-time signature along with the verification key $Y_{s}$.

In order to verify the signature, the verifier performs a standard one-time signature verification using the verification key $Y_{s}$, and then uses Merkle tree in order to verify $Y_{s}$. To assist the verifier in this process, the signer publishes the verification path, $V=\left(a_{0}, \ldots, a_{\ell-1}\right)$, where each $a_{h}$ corresponds to the sibling of the height- $h$ node along the path from leaf $H\left(Y_{S}\right)$ to the Merkle tree root node. With this information at hand, the verifier can reproduce the computations in Eq. (1), traversing the tree upwards to the root; the verification of $Y_{s}$ is successful if the final result matches the Merkle tree public key, $n_{\ell}[0]$.

Fig. 1 Merkle tree for $\ell=3$

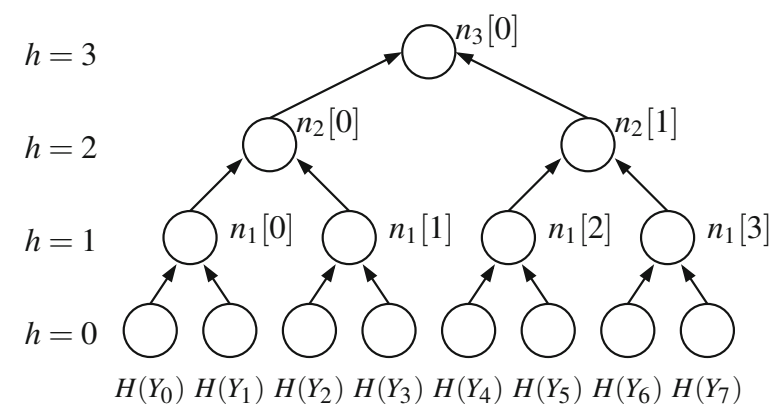




\subsection{Code-Based Cryptography}

The first code-based proposals can be traced back to McEliece's in 1978 [13]. The idea behind this and other similar encryption schemes stems from the world of electronic transmissions over noisy channels: most of the time the receiver is expected to receive a distorted signal, so the sender adds some extra information that depends on the data transmitted, allowing the receiver to reconstruct the original data even in the presence of transmission errors (up to a certain level).

Assuming that we transmit bits, this strategy ensures that not any bit pattern but only specific ones, termed codewords, are valid. These codewords are a certain "distance" apart in the code space so that any received bit pattern is either a valid codeword or can be "corrected" to a valid one, as long as the error remains below a certain bound. Often, Hamming distance is chosen, whereby two $n$-bit strings are at a Hamming distance of $t$ if they differ precisely in $t$ bits.

Translating these ideas to the cryptographic world, one can think of an encryption scheme such that a sender "disguises" the information by "adding" some noise, which only the legitimate receiver is able to prune since she is in possession of the "key", namely the right "correcting code."

The "workhorse" for a McEliece-like cryptosystem is, therefore, a (linear) correction code, $\mathcal{C}$, able to correct up to $t$ bits in error, and possessing an efficient decoding algorithm. We assume in the sequel that we are provided with such a code, represented by a $k \times n$ matrix $G$, and the base field is $\mathbb{F}_{2}$.

The user $A$ generates her public/private key pair as follows:

1. A chooses a matrix $S \in G L\left(n, \mathbb{F}_{2}\right)$ with entries at random.

2. $A$ chooses at random a $k \times k$ permutation matrix $P$.

3. A computes the $k \times n$ matrix $H=P G S$.

4. A publishes $(H, t)$ as her public key. She keeps $(S, G, P)$ as her private key.

If a user $B$ is willing to send a message $m$ to $A$, she takes the following steps:

1. $B$ encodes the message $m$ as an $n$-bit string.

2. $B$ computes $c^{*}=H m$.

3. $B$ generates at random $z \in \mathbb{F}_{2}^{k}$ with weight $t$ (i.e., with exactly $t$ bits set to 1 ).

4. $B$ computes and sends to $A$ the ciphertext $c=c^{*}+z$.

Remark that $z$ plays the role of "adding errors" along the transmission, transforming $c$ * into a "distorted" value $c$.

Upon receipt of $c, A$ takes the following steps to recover the message:

1. $A$ computes $c^{\prime}=P^{-1} c$.

2. $A$ uses the decoding algorithm to decode $c^{\prime}$ to $m^{\prime}$.

3. $A$ recovers $m=S^{-1} m^{\prime}$. 
We can check that the deciphering is correct since

$$
\begin{aligned}
c^{\prime}= & P^{-1} c=P^{-1} c^{*}+P^{-1} z=P^{-1} H m+P^{-1} z \\
& =P^{-1} P G S m+P^{-1} z=G S m+P^{-1} z .
\end{aligned}
$$

The last term means that the decoding algorithm returns $m^{\prime}=S m$, discarding the "noise" represented by $P^{-1} z$, whence $m=S^{-1} m^{\prime}$.

\subsection{Lattice-Based Cryptography}

Given a set $\mathbf{B}=\left\{\mathbf{b}_{1}, \ldots, \mathbf{b}_{n}\right\}, \mathbf{b}_{i} \in \mathbb{R}^{n}$, of linearly independent vectors, a lattice $\mathcal{L}$, is defined as the set

$$
\mathcal{L}\left(\mathbf{b}_{1}, \ldots, \mathbf{b}_{n}\right)=\left\{\sum_{i=1}^{n} z_{i} \mathbf{b}_{i}: z_{i} \in \mathbb{Z}\right\}
$$

Under this definition, $\mathcal{L} \subset \mathbb{R}^{n}$ has the structure of a discrete additive subgroup of $\mathbb{R}^{n}$. We consider the standard inner product in $\mathbb{R}^{n}$ and the norm of any vector $\mathbf{x}$ as the standard $L_{2}$ Euclidean norm, $\|\mathbf{x}\|^{2}=\langle\mathbf{x}, \mathbf{x}\rangle$. The set $\mathbf{B}$ is called a basis for the lattice $\mathcal{L}$, though any lattice admits multiple bases: for example, if $\mathbf{U}$ is a unimodular matrix, then $\mathcal{L}(\mathbf{B})=\mathcal{L}(\mathbf{U B})$. In particular, the determinant of the matrix representing the base $\mathbf{B}$ is independent of the choice of $\mathbf{B}$. The seminal work of Ajtai [14] set the foundations of lattice-based cryptography defining the first two problems of those described below.

Among the best-known lattice-related problems that give rise to cryptographic applications, we can find:

1. SVP (shortest-vector problem): given a lattice, $\mathcal{L}(\mathbf{B})$, find the shortest (non-zero) vector in it.

2. CVP (closest-vector problem): given a lattice, $\mathcal{L}(\mathbf{B})$, and a target vector $\mathbf{t} \in \mathbb{R}^{n}$, find the vector $\mathbf{v} \in \mathcal{L}(\mathbf{B})$ closest to $\mathbf{t}$ according to the selected $L_{2}$ norm.

3. SIS (short-integer-solution problem): Let $n$ and $q$ be integers ( $n$ is a security parameter, and usually $q=\operatorname{poly}(n))$, and let $\beta>0$. Given a uniformly random matrix $A \in \mathbb{Z}_{q}^{n \times m}$ for some $m=\operatorname{poly}(n)$, find a (non-zero) integer vector $\mathbf{z} \in$ $\mathbb{Z}^{m}$, such that $A \mathbf{z}=\mathbf{0}(\bmod q)$ and $\|\mathbf{z}\| \leq \beta$, using $L_{2}$ norm. Remark that $\beta$ must be large enough to ensure that a solution does exist, but $\beta>q$ makes the problem trivially easy to solve. Ajtai [14] showed that for appropriate parameters, solving SIS on the average is (with non-negligible probability) at least as hard as approximating several lattice problems on $n$-dimensional lattices in the worst case (up to $\operatorname{poly}(n)$ factors). 
4. LWE (learning-with-errors problem): Given parameters $n$ and $q$ as before, and a "noise rate," $\alpha$, we choose $\mathbf{s} \in \mathbb{Z}_{q}^{n}$ and form pairs $\left(\mathbf{a}_{i}, b_{i}=\left\langle\mathbf{a}_{i}, \mathbf{s}\right\rangle+e_{i}\right) \in$ $\mathbb{Z}_{q}^{n} \times \mathbb{Z}_{q}$, where each $\mathbf{a}_{i}$ is uniformly randomly selected, and $e_{i}$ is picked from $\mathbb{Z}_{q}^{m}$ following a Gaussian-like distribution $\chi: \mathbb{Z}_{q} \rightarrow \mathbb{R} / \mathbb{Z}$ with standard deviation roughly equal to $\alpha q$. The LWE problem (in its "search" version) consists in finding the secret vector $\mathbf{s} \in \mathbb{Z}_{q}^{n}$. This problem was posed by Regev [15] who showed that for certain choices of $q$ and $\chi$, solving LWE on the average is (with non-negligible probability) at least as hard as approximating lattice problems in the worst case to within $\tilde{O}(n / \alpha)$ factors using a quantum algorithm. Later on, similar results were proved for classical algorithms [16].

There also exists the "decision" version of the LWE problem, whereby given pairs $\left(\mathbf{a}_{i}, b_{i}\right)$, the target is deciding whether they have been generated as above or they are truly random.

The problem can be used to define a public key cryptosystem. The user $A$ selects a vector $\mathbf{s} \in \mathbb{Z}_{q}^{n}$ as private key. She also picks random $m$ vectors $\mathbf{a}_{i} \in \mathbb{Z}_{q}^{n}$, and $m$ values $e_{i} \in \mathbb{Z}_{q}$ according to error distribution $\chi$. She publishes the $m$ pairs $\left(\mathbf{a}_{i}, b_{i}\right)$, with $b_{i}=\left\langle\mathbf{a}_{i}, b_{i}\right\rangle+e_{i}$, as her public key. If a user $B$ is willing to send a ciphered bit to $A$, first selects a random subset $S$ from the power set of $\{1, \ldots, m\}$. The encryption is defined as $(\overline{\mathbf{a}}, \bar{b})=\left(\sum_{i \in S} \mathbf{a}_{i}, \sum_{i \in S} b_{i}\right)$ if the bit to be encrypted is 0 ; and $(\overline{\mathbf{a}}, \bar{b})=\left(\sum_{i \in S} \mathbf{a}_{i},\lfloor q / 2\rfloor+\sum_{i \in S} b_{i}\right)$ otherwise.

To decipher, user $A$ simply computes $\bar{b}-\langle\overline{\mathbf{a}}, \mathbf{s}\rangle$, and checks whether it is nearer to 0 (then the decrypted bit is 0 ) or to $\lfloor q / 2\rfloor$ (the decrypted bit is 1 ).

These descriptions follow closely [17].

5. R-LWE (ring-learning-with-errors problem): It is a variant of the previous LWE aiming at optimizing the efficiency in terms of key length and speed of operation. We give hereafter a simplified version of this problem, following [18]. Let $f(x)=x^{n}+1 \in \mathbb{Z}[x]$, with $n$ a power of 2 , an irreducible polynomial over the rationals. We consider the ring $R=\mathbb{Z}[x] /\langle f(x)\rangle$, the ring of integer polynomials modulo the ideal generated by $f(x)$. Technically, given $m=2^{k}$, then $f(x)$ is the $m$-th cyclotomic polynomial, which is of degree $\varphi(m)=2^{k-1}=n$, and $R$ can be seen as the ring of integers of the algebraic number field $\mathbb{Q}\left(\zeta_{m}\right)$, where $\zeta_{m}$ is a primitive $m$-root of the unity ( $\zeta_{m}$ and its powers co-prime to $m$ are precisely the roots of $f(x))$. Let $q$ be a sufficiently large prime number such that $q \equiv 1$ $\left(\bmod m\right.$ ). We now define $R_{q}=R /\langle q\rangle$, namely the finite ring of polynomials with integer coefficients modulo $q$ and degree less than $n$.

Fixing now a certain probability (error) distribution $\chi$ over $R$ concentrated on "small" elements of $R$ (informally, those with small integer coefficients) and randomly selecting an element $s \in R_{q}$ form a number of pairs $\left(a_{i}, b_{i}=a_{i} \cdot s+e_{i}\right)$, where each $a_{i}$ has been uniformly randomly selected from $R_{q}$ and each $e_{i}$ has been drawn according to the error distribution $\chi$. Given the pairs $\left(a_{i}, b_{i}\right)$, the "search" version of the R-LWE problem consists in finding the secret element $s$. The "decision" problem consists in distinguishing pairs generated as above from truly random uniform pairs.

The gain in efficiency with the variant R-LWE is twofold. First, the product $b=$ $a \cdot s+e$ gives simultaneously $n$ values over $\mathbb{Z}_{q}$, where the LWE gives only a 
scalar, while the cost is much smaller by using FFT multiplication techniques. Second, each pair $\left(a_{i}, b_{i}\right) \in R_{q} \times R_{q}$ can replace $n$ samples $\left(\mathbf{a}_{i}, b_{i}\right) \in \mathbb{Z}_{q}^{n} \times \mathbb{Z}_{q}$, which results in an $n$-fold saving in the size of the public key.

To encrypt a message $m \in\{0,1\}^{n}$, we embed it in $R$ via "coefficient embedding." Then we draw "small" elements $r, e_{1}, e_{2} \in R$ with the error distribution and consider the ciphertext as the pair $(u, v) \in R \times R$ given by

$$
u=a \cdot r+e_{1} \quad \text { and } \quad v=b \cdot r+e_{2}+\lfloor q / 2\rceil \cdot m .
$$

To decipher, the legitimate user computes

$$
v-u \cdot s=\left(r \cdot e-s \cdot e_{1}+e_{2}\right)+\lfloor q / 2\rceil \cdot m .
$$

If the parameters of the system are wisely selected, the coefficients of $r \cdot e-s$. $e_{1}+e_{2}$ are smaller than $q / 4$ so the bits of $m$ can be recovered rounding each coefficient in $v-u \cdot s$ to either 0 or $\lfloor q / 2\rceil$, whichever is closer.

The interesting point is that all the previous described problems are proved to be $\mathcal{N P}$-hard.

\subsection{Multivariate-Quadratic-Equations Cryptography}

Another vein of hard problems stems from the world of Algebraic Geometry and gives rise to the so-called Multivariate Quadratic Cryptography. Introduced by Matsumoto and Imai in 1988 [19], it is based on the difficulty of the $\mathcal{M Q}$ problem.

The $\mathcal{M Q}$ problem over a finite field of $q$ elements $\mathbb{F}_{q}$ consists in finding a solution $\mathbf{x} \in \mathbb{F}_{q}^{n}$ to a given system of $m$ quadratic polynomial equations $\mathbf{p}=$ $\left(p_{1}, \ldots, p_{m}\right)$ over $\mathbb{F}_{q}$ in $n$ indeterminates, where each polynomial $p_{k}$ is of the form:

$$
p_{k}\left(x_{1}, \ldots, x_{n}\right)=\sum_{i \geq j} a_{i j k} x_{i} x_{j}+\sum_{i} b_{i k} x_{i}+c_{k},
$$

for $1 \leq k \leq m$, and all the coefficients $a_{i j k}, b_{i k}, c_{k}$, in $\mathbb{F}_{q}$.

This problem is $\mathcal{N} \mathcal{P}$-complete over any finite field for a randomly selected polynomial vector $\mathbf{p}=\left(p_{1}, \ldots, p_{m}\right)([20, \mathrm{p} .251]$; [21, section 2.5]; [22]). However, a real-life cryptosystem needs some kind of structure in order to implement the "trap-door" but even in this case, current research seemingly suggests that $\mathcal{M Q}$ problem is also hard on average (see [23], [24]).

$\mathcal{M Q}$ problem gives rise both to encryption and signature schemes. A simple encryption scheme works based upon a central map, an easy-to-invert quadratic function $\mathcal{Q}: \mathbb{F}_{q}^{n} \rightarrow \mathbb{F}_{q}^{m}$, which plays the role of a private key. In order to hide the central map, we choose two (usually affine) invertible transformations, $S: \mathbb{F}_{q}^{n} \rightarrow \mathbb{F}_{q}^{n}$ 
Fig. 2 The (blue) public and the (red) private "routes"

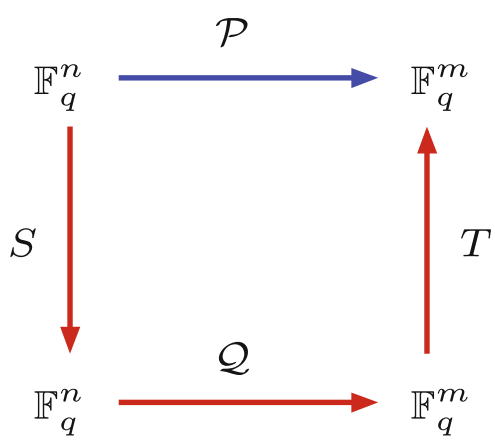

and $T: \mathbb{F}_{q}^{m} \rightarrow \mathbb{F}_{q}^{m}$. The public key is the composition of those maps, namely $\mathcal{P}=$ $T \circ \mathcal{Q} \circ S$. Figure 2 depicts the composition.

The ciphering process consists in evaluating the polynomial $\mathcal{P}$ over a vector $\mathbf{m}$ that represents the message in order to get the ciphertext $\mathbf{c}=\mathcal{P}(\mathbf{m})$. An adversary willing to break the system must needs solve an instance of the $\mathcal{M Q}$ problem to recover the message. For the legitimate receiver this task is very easy, since she knows the secret key (the decomposition of $\mathcal{P}$ ) so she can compute $S^{-1}, T^{-1}$, and $\mathcal{Q}^{-1}$, and carry out the deciphering process:

$$
\begin{gathered}
\left(S^{-1} \circ \mathcal{Q}^{-1} \circ T^{-1}\right)(\mathbf{c})=\left(S^{-1} \circ \mathcal{Q}^{-1} \circ T^{-1} \circ \mathcal{P}\right)(\mathbf{m}) \\
=\left(S^{-1} \circ \mathcal{Q}^{-1} \circ T^{-1} \circ T \circ \mathcal{Q} \circ S\right)(\mathbf{m})=\mathbf{m} .
\end{gathered}
$$

A simple signature scheme is also possible: Consider a hash function $H:\{0,1\}^{*} \rightarrow \mathbb{F}_{q}^{m}$, and let $A$ be a user with public key $\mathcal{P}$ and corresponding private key $(S, \mathcal{Q}, T)$. Assume that $A$ is willing to sign a message $\mathbf{m}$ : Then $A$ first computes $h=H(\mathbf{m}) \in \mathbb{F}_{q}^{m}$, and obtains the signature $\sigma$ by applying the composition $\left(S^{-1} \circ \mathcal{Q}^{-1} \circ T^{-1}\right)(h)=\sigma \in \mathbb{F}_{q}^{n}$.

Suppose a verifier is given the pair $(\mathbf{m}, \sigma)$. The verification process consists of applying the public key to $\sigma$, thus obtaining $\bar{h}=\mathcal{P}(\sigma)$. The verifier considers the signature valid if and only if $\bar{h}=H(\mathbf{m})$.

\subsection{Elliptic-Curve-Isogeny-Based Cryptography}

The core of this problem and its application to cryptography is rather involved, and its description would require too much space. We content ourselves with citing it in the context of the class of problems that are seemingly quantum-resistant. 


\section{Chor-Rivest Knapsack Cryptosystem}

Chor and Rivest proposed a cryptosystem based on knapsack problem and on arithmetic in finite fields [7]. It needs to compute discrete logarithms in order to determine the keys of the system. One of the advantages of this cryptosystem is that the Chor-Rivest knapsack density is greater than 1. This made difficult low density-based attacks [25]. Although the system was broken for some specific parameters [26] enabling the recovery of the private key from the public one, it remains unbroken for some other parameters. The knapsack problem is considered a "decision" $\mathcal{N} \mathcal{P}$-complete problem [27].

For the sake of brevity, we will denote Chor-Rivest cryptosystem simply as CRC.

\subsection{Key Generation System}

To define the C-RC, the following parameters must be considered (see [7]):

- The power of a primer number, $q=p^{\lambda}$, and a positive integer $h \leq q$ such that the calculation of logarithms in $\mathbb{F}_{q^{h}}$ can be efficiently carried out.

- A root $t \in \mathbb{F}_{q^{h}}$ from an irreducible monic polynomial of degree $h, F(x) \in \mathbb{F}_{q}[x]$. Any element of $\mathbb{F}_{q^{h}}$ can be represented as a polynomial in $t$ with degree $\leq h$ and coefficients in $\mathbb{F}_{q}$.

- A generator, $g$, of the multiplicative group $\mathbb{F}_{q^{*}}^{*}$. Observe, in passing, that the order of $\mathbb{F}_{q^{h}}^{*}$ is $n=q^{h}-1$.

- The following $q$ logarithms that are to be computed:

$$
a_{i}=\log _{g}\left(t+\alpha_{i}\right),
$$

for all $\alpha_{i} \in \mathbb{F}_{q}, 0 \leq i \leq q-1$.

- The elements $a_{i}$ are reordered by a random permutation

$$
\pi:\{0,1, \ldots, q-1\} \rightarrow\{0,1, \ldots, q-1\},
$$

in such a way that $b_{i}=a_{\pi(i)}$.

- A noise is added, considering a randomly chosen integer number $0 \leq r \leq q^{h}-2$, and then

$$
c_{i} \equiv\left(b_{i}+r\right) \quad\left(\bmod q^{h}-1\right), \quad i=0,1, \ldots, q-1 .
$$

- The user's public key is the set $\left\{c_{0}, c_{1}, \ldots, c_{q-1}, q, h\right\}$.

- The user's private key is the set $\{t, g, \pi, r\}$. 
Thus, the key size in this cryptosystem is dependent on the size of the parameters originally considered.

\subsection{Encryption Process}

To cipher a message, $M$, it must first be converted into a vector of $q$ bits with weight $h$, namely with exactly $h$ bits set to 1 ,

$$
M=\left(x_{0}, x_{1}, \ldots, x_{q-1}\right), \quad x_{i} \in\{0,1\}, \quad i=0, \ldots, q-1 .
$$

The ciphertext, $C$, corresponding to $M$ is calculated by adding the values $c_{i}$ for which $x_{i}=1$, i.e.,

$$
C=\sum_{i=0}^{q-1} x_{i} \cdot c_{i}\left(\bmod q^{h}-1\right) .
$$

\subsection{Decryption Process}

In order to decrypt a ciphertext $C$, assuming that it comes from a valid $q$-bit message with weight $h$, the following steps are considered:

1. Compute $C^{\prime} \equiv C-h \cdot r\left(\bmod q^{h}-1\right)$.

2. Obtain $g^{C^{\prime}}$ written as a polynomial in $x$. There exists a unique polynomial $Q(x) \in \mathbb{F}_{q^{h}}[x]$ with degree $\leq h-1$, such that

$$
Q(x) \equiv g^{C^{\prime}}(\bmod F(x)) .
$$

Naming as $I=\left\{i_{1}<\cdots<i_{h}\right\}$ the set of indices with corresponding bits equal to 1 , i.e., $x_{i_{1}}=\cdots=x_{i_{h}}=1$, then:

$$
\begin{aligned}
g^{C^{\prime}} & =g^{C} \cdot g^{-h \cdot r}=g^{\sum_{i=0}^{q-1} x_{i} \cdot c_{i}} \cdot g^{-h \cdot r}=\prod_{i \in I} g^{c_{i}-r} \\
& =\prod_{i \in I} g^{b_{i}}=\prod_{i \in I} g^{a_{\pi(i)}}=\prod_{i \in I}\left(t+\alpha_{\pi(i)}\right)
\end{aligned}
$$

3. As the polynomial with degree $h, F(x)+Q(x)$ factorizes linearly in the field $\mathbb{F}_{q}$, then 


$$
F(x)+Q(x)=\prod_{i \in I}\left(x+\alpha_{\pi(i)}\right) .
$$

Replacing all values $\alpha_{0}, \alpha_{1}, \ldots, \alpha_{q-1} \in \mathbb{F}_{q}$, the $h$ roots of that polynomial are obtained. If those roots are $\alpha_{j_{1}}, \ldots, \alpha_{j_{h}}$, then applying the inverse permutation $\pi^{-1}$ to those roots indices, $\pi^{-1}\left(j_{l}\right)=i_{l}$, subindices of the message with terms equal to 1 are obtained.

\subsection{Safe Parameters}

Several attacks are known to break C-RC but, in practice, they succeed only for the system parameters originally proposed by the authors in 1988 . We mention some of them hereafter.

Chor and Rivest [7] describe both specialized attacks, where the attacker knows parts of the recipient's secret key, such as the one designed by Goldreich and Odlyzko; and general attacks, where only the public key is available. Among the latter, we can count the brute-force attack designed by Brickell [28], low-density knapsack attacks such as the one performed by Lagarias and Odlyzko [25], and others such as the one by Schnorr and Hörner [29].

The pairs of parameters $(q, h)$ originally proposed for C-RC by Chor and Rivest were $(197,24),(211,24),\left(3^{5}, 24\right)$, and $\left(2^{8}, 25\right)$, whose number of digits are, respectively, 56, 56, 58, and 60 . If the number of digits is small enough, as is the case for the pairs $(103,12)$ and $(151,16)$, the Schnorr-Hörner attack is capable of breaking the cryptosystem [29].

In 2001, Vaudenay [8] was the first to really break C-RC under a much broader range of parameters (including those proposed by Chor and Rivest). Vaudenay considers the finite field $\mathbb{F}_{q^{h}}, q$ being a prime or the power of a prime number, and $h$ an integer. His attack is remarkable since he exploits the fact that this C-RC has equivalent secret keys, and he is able to recover one of them. An equivalent key is (in general) different than the original secret key, but it works the same as it were the right one. All users can use the same values for $q$ and $h$ because the risk of collisions (that is, that two users have the same password) is extremely small. As the private key is made up of the set $\{t, g, \pi, r\}$, there are

$$
h \cdot \phi\left(q^{h}-1\right) \cdot\left(q^{h}-2\right) \cdot q !
$$

different private keys, from which $h \cdot q(q-1)$ are equivalent, where $\phi$ is the Euler's indicator. Then, the number of non-equivalent keys is

$$
n_{q, h}=\phi\left(q^{h}-1\right) \cdot\left(q^{h}-2\right) \cdot(q-2) !
$$

Hence, for a set of $k$ users the number of possible collisions will be 


$$
\frac{\left(n_{q, h}-1\right)\left(n_{q, h}-2\right) \cdots\left(n_{q, h}-k+1\right)}{n_{q, h}^{k}},
$$

which is a small number, given that $1 / n_{q, h}$ is an upper bound of that value. As an example, for the pair of parameters $q=197, h=24$, the bound could be calculated as

$$
\frac{\left(n_{q, h}-1\right)\left(n_{q, h}-2\right) \cdots\left(n_{q, h}-k+1\right)}{n_{q, h}^{k}}<0.16155 \cdot 10^{-472} .
$$

However, all the attacks just described are able to break the C-RC only if the original parameters (remember they date back to 1988!) or parameters showing certain properties (in the case of Vaudenay's) are used. This opens the vein of exploring either parameters lying in broader ranges or parameters selected to circumvent the known attacks.

In $[9,10]$ it is proven that several pairs of parameters $(q, h)$ could be found verifying the conditions established by $\mathrm{C}-\mathrm{RC}$ and making the cryptosystem strong against the known attacks, namely:

1. $2 \leq h \leq q$, with $q$ a prime number, and $h$ a prime number or the square of a prime number,

2. the number of digits of $n$ is $t(q, h) \geq 36$,

3 . the bitlength of the public key satisfies $l(q, h)<15,000$,

4. the density of the knapsack, $d(q, h)$, is greater than 1 , and

5. the smoothness of $n, u(n)$, has at most 18 decimal digits.

The pairs verifying these conditions are included in Table 1. Remark that the pair $(1123,13)$ exhibits the longest public key size, which is on the order of 150 kilobits. Such a size could be deemed too large in 2008 or 2009, but this is no longer the case: It suffices to observe that the key sizes of the proposals presented to the second round of the NIST call [5] are on the order of several megabytes.

\subsection{Experimental Results}

We have conducted an experiment in order to assess the computation time required to perform an encryption and a subsequent decryption of messages with various representative bit sizes, and system parameters.

We implemented the protocol with Magma computer algebra system [30], in particular using its language with version Magma V2.20-10. The Magma programs were run over an Intel Core i $7 / 860$ at $2.80 \mathrm{GHz}$ with $12 \mathrm{~GB}$ of RAM under Windows 10 Pro using a single running thread.

We chose three message sizes: a "small-sized" message, with about $1 \mathrm{~KB}$; a "medium-sized" message, with about $200 \mathrm{~KB}$; and finally a "large-sized" message, 
Table 1 Values of the pairs $(q, h)$ verifying the conditions (1)-(5)

\begin{tabular}{l|l|l|l|l|l}
\hline$h$ & $q$ & $t(q, h)$ & $l(q, h)$ & $d(q, h)$ & $u(n)$ \\
\hline 13 & 547 & 36 & 64,546 & 4.626 & 14 \\
\hline 13 & 571 & 36 & 67,949 & 4.796 & 13 \\
\hline 13 & 577 & 36 & 68,663 & 4.838 & 14 \\
\hline 13 & 599 & 37 & 71,281 & 4.994 & 13 \\
\hline 13 & 601 & 37 & 72,120 & 5.008 & 11 \\
\hline 13 & 613 & 37 & 73,560 & 5.092 & 15 \\
\hline 13 & 631 & 37 & 75,720 & 5.218 & 11 \\
\hline 13 & 641 & 37 & 77,561 & 5.288 & 17 \\
\hline 13 & 659 & 37 & 79,739 & 5.413 & 16 \\
\hline 13 & 683 & 37 & 83,326 & 5.579 & 14 \\
\hline 13 & 719 & 38 & 88,437 & 5.828 & 15 \\
\hline 13 & 757 & 38 & 93,868 & 6.088 & 16 \\
\hline 13 & 787 & 38 & 98,375 & 6.292 & 15 \\
\hline 13 & 797 & 38 & 99,625 & 6.360 & 13 \\
\hline 13 & 839 & 39 & 105,714 & 6.644 & 11 \\
\hline 13 & 877 & 39 & 111,379 & 6.900 & 16 \\
\hline 13 & 887 & 39 & 112,649 & 6.967 & 16 \\
\hline 13 & 941 & 39 & 120,448 & 7.327 & 14 \\
\hline 13 & 953 & 39 & 121,984 & 7.407 & 16 \\
\hline 13 & 967 & 39 & 123,776 & 7.500 & 14 \\
\hline 13 & 977 & 39 & 126,033 & 7.566 & 16 \\
\hline 13 & 1093 & 40 & 143,183 & 8.329 & 13 \\
\hline
\end{tabular}

\begin{tabular}{l|l|l|l|l|l}
\hline$h$ & $q$ & $t(q, h)$ & $l(q, h)$ & $d(q, h)$ & $u(n)$ \\
\hline 13 & 1123 & 40 & 147,113 & 8.524 & 16 \\
\hline 17 & 127 & 36 & 14,986 & 1.068 & 15 \\
\hline 17 & 131 & 36 & 15,589 & 1.095 & 15 \\
\hline 17 & 167 & 38 & 20,875 & 1.330 & 14 \\
\hline 17 & 193 & 39 & 24,897 & 1.495 & 17 \\
\hline 17 & 233 & 41 & 30,989 & 1.742 & 15 \\
\hline 17 & 263 & 42 & 35,768 & 1.924 & 15 \\
\hline 17 & 277 & 42 & 37,949 & 2.008 & 15 \\
\hline 17 & 317 & 43 & 44,697 & 2.244 & 17 \\
\hline 17 & 331 & 43 & 47,002 & 2.326 & 17 \\
\hline 17 & 409 & 45 & 60,123 & 2.773 & 9 \\
\hline 17 & 433 & 45 & 64,084 & 2.908 & 18 \\
\hline 17 & 587 & 48 & 91,572 & 3.754 & 14 \\
\hline 17 & 643 & 48 & 101,594 & 4.054 & 17 \\
\hline 17 & 661 & 48 & 105,099 & 4.150 & 16 \\
\hline 23 & 173 & 52 & 29,410 & 1.011 & 18 \\
\hline 23 & 191 & 53 & 33,234 & 1.095 & 18 \\
\hline 23 & 199 & 53 & 34,825 & 1.132 & 15 \\
\hline 23 & 283 & 57 & 52,921 & 1.510 & 16 \\
\hline 23 & 563 & 64 & 118,230 & 2.679 & 14 \\
\hline 25 & 601 & 70 & 138,230 & 2.604 & 17 \\
\hline 25 & 613 & 70 & 141,603 & 2.648 & 16 \\
\hline & & & & & \\
\hline 13
\end{tabular}

Table 2 Encryption/decryption times for various $(q, h)$ and message sizes

\begin{tabular}{l|l|c|c|c|c}
\hline Size & Action & $(167,17)$ & $(409,17)$ & $(631,13)$ & $(839,13)$ \\
\hline $1 \mathrm{~KB}$ & Enc time & 0.042 & 0.075 & 0.090 & 0.120 \\
\hline $1 \mathrm{~KB}$ & Dec time & 0.153 & 0.138 & 0.106 & 0.109 \\
\hline $200 \mathrm{~KB}$ & Enc time & 8.636 & 12.547 & 16.975 & 23.655 \\
\hline $200 \mathrm{~KB}$ & Dec time & 30.858 & 25.048 & 23.019 & 24.878 \\
\hline $1 \mathrm{MB}$ & Enc time & 52.895 & 68.667 & 106.403 & 147.969 \\
\hline $1 \mathrm{MB}$ & Dec time & 185.998 & 153.794 & 147.488 & 158.383
\end{tabular}

with some $1 \mathrm{MB}$. In most common situations, one would use a "small-sized" message in order to setup a key exchange (for example, to implement a key encapsulation mechanism). We selected four different configuration for the system parameters $(q, h)$.

Table 2 summarizes the results: The computation times are measured in seconds. Each pair of lines represents the encryption time (Enc time) and decryption time (Dec time) for a particular message size and four different system parameters: $(167,17),(409,17),(631,13)$, and $(839,13)$. The interesting point is that for the "small-sized" message, both encryption and decryption times are on the order of 
one tenth of a second, whereas the system remains of little use for "large-sized" messages.

\section{Discussion and Future Work}

Modern advances in quantum computers pose a looming threat to current cryptosystems, those based on (classically) intractable problems, since quantum algorithms do exist that solve in polynomial time ( $=$ fast) those problems. This fact has triggered the interest and research towards quantum-resistant problems that could act as a "drop-in replacement" to safeguard the security and strength of cryptography.

In this paper, we have introduced some of the problems currently considered as quantum-resistant, which make up the building blocks of the "Post-Quantum Cryptography."

However, we feel that in this new scenario C-RC has not received proper attention and its security has not been analyzed in due detail. For one thing, the mathematical problems in which C-RC are based upon have not been considered by the NIST Call in [4]. It is true that the majority, if not all, knapsack-type cryptosystems has been broken at some point, a fact that may account for the distrust currently elicited by this type of cryptosystems.

The interesting point is that $\mathrm{C}-\mathrm{RC}$ is a knapsack-type cryptosystem whose security is not based upon discrete logarithms, even though it needs to compute them in order to set up the system. Actually, any reduction in the computation time of such logarithms means an advantage for $\mathrm{C}-\mathrm{RC}$, since both the public and the private keys could be obtained in a much shorter time-frame. Still better, we are allowed to use much longer (thus safer) key sizes if the advent of the universal quantum computer is ever realized: Shor's algorithm [1] would permit users to reach key sizes not dreamed of when the system was originally proposed. In fact, one of its drawbacks was precisely the necessity of computing such discrete logarithms, for which the only known "efficient" algorithms were "Baby step-Giant step," or Pohlig-Hellman's [27]. Since these algorithms exhibit a subexponential running time, the use of long and safe key lengths was precluded in practice. All these facts may very well make C-RC deserve a proper place at the post-quantum candidates dinner table. In view of the latter, we suggest several research lines to be considered:

- To increase the key sizes in knapsack-like cryptosystems so as to be safe in the face of quantum computation while keeping efficiency in the key generation process.

- Analyze the security of this cryptosystem endowed with new, revamped parameter sizes in front of the threat posed by quantum computers.

- Improve the key generation process so as to thwart attacks based on equivalent keys (such as Vaudenay's) even in the presence of quantum computers. 
Acknowledgments This work has been partially supported by Ministerio de Economía, Industria y Competitividad (MINECO), Agencia Estatal de Investigación (AEI), and European Regional Development Fund (ERDF), through project COPCIS, grant no. TIN2017-84844-C2-1-R, and by Comunidad de Madrid (Spain) through project CYNAMON, grant no. P2018/TCS-4566-CM, cofunded with ERDF.

\section{References}

1. P. Shor, Polynomial-time algorithms for prime factorization and discrete logarithms on a quantum computer. SIAM J. Comput. 26, 1484-1509 (1997)

2. L. Grover, Quantum mechanics helps in searching for a needle in a haystack. Phys. Rev. Lett. 79, 325-328 (1997)

3. D. Simon, On the power of quantum computation. SIAM J. Comput. 26(5), 1474-1483 (1997)

4. NIST, Post-quantum cryptography. On-line publication. 2017. https://csrc.nist.gov/Projects/ Post-Quantum-Cryptography

5. NIST, Post-quantum cryptography, 2nd round. On-line publication, 2019. https://csrc.nist.gov/ projects/post-quantum-cryptography/round-2-submissions

6. B.-Z. Chor, Two Issues in Public Key Cryptography. RSA Bit Security and a New Knapsack Type System. ACM Distinguished Dissertation. The MIT Press, Cambridge, MS (1986)

7. B. Chor, R. Rivest, A knapsack-type public key cryptosystem based on arithmetic in finite fields. IEEE Trans. Inform. Theor. 34(5), 901-909 (1988)

8. S. Vaudenay, Cryptanalysis of the Chor-Rivest cryptosystem. J. Cryptol. 14, 87-100 (2001)

9. L. Hernández Encinas, J. Muñoz Masqué, A. Queiruga Dios, Safer parameters for the ChorRivest cryptosystem. Comput. Math. Appl. 56, 2883-2886 (2008)

10. L. Hernández Encinas, J. Muñoz Masqué, A. Queiruga Dios, Analysis of the efficiency of the Chor-Rivest cryptosystem implementation in a safe-parameter range. Inf. Sci. 179, 4219-4226 (2009)

11. D. Bernstein, J. Buchmann, E. Dahmen (eds.), Post-quantum Cryptography (Springer, Berlin, Heidelberg, 2009)

12. L. Lamport, Constructing digital signatures from a one way function. SRI International Computer Science Laboratory, Technical Report SRI-CSL-98 (1979)

13. R.J. McEliece, A public-key cryptosystem based on algebraic coding theory. Jet Propulsion Laboratory, Technical Report 42-44 (1978)

14. M. Ajtai, Generating hard instances of lattice problems (extended abstract), in Proceedings of the Twenty-Eighth Annual ACM Symposium on Theory of Computing, STOC '96 (Association for Computing Machinery, New York, NY, 1996), pp. 99-108

15. O. Regev, On lattices, learning with errors, random linear codes, and cryptography. J. ACM 56(6), 1-40 (2009). Art. no. 34

16. C. Peikert, Public-key cryptosystems from the worst-case shortest vector problem: extended abstract, in Proceedings of the Forty-First Annual ACM Symposium on Theory of Computing, STOC '09 (Association for Computing Machinery, New York, NY, 2009), pp. 333-342

17. D. Micciancio, C. Peikert, Hardness of SIS and LWE with small parameters, in Advances in Cryptology - CRYPTO 2013, ed. by R. Canetti, J.A. Garay (Springer, Berlin, Heidelberg, 2013), pp. 21-39

18. V. Lyubashevsky, C. Peikert, O. Regev, On ideal lattices and learning with errors over rings. J. ACM 60(6), 1-35 (2013)

19. T. Matsumoto, H. Imai, Public quadratic polynomial-tuples for efficient signature-verification and message-encryption. Lect. Notes Comput. Sci. 330, 19-453 (1988)

20. M.R. Garey, D.S. Johnson, Computer and Intractability: A Guide to the Theory of NPCompleteness (W. H. Freeman \& Co, New York, 1990) 
21. C. Wolf, Multivariate quadratic polynomials in public key cryptography, Ph.D. dissertation, Katholieke Universiteit Leuven, November (2005)

22. J. Patarin, Cryptanalysis of the Matsumoto and Imai public key scheme of Eurocrypt ' 88 . Lect. Notes Comput. Sci. 963, 248-261 (1995)

23. N. Courtois, A. Klimov, J. Patarin, A. Shamir, Efficient algorithms for solving overdefined systems of multivariate polynomial equations. Lect. Notes Comput. Sci. 1807, 392-407 (2000)

24. N. Courtois, L. Goubin, W. Meier, J.-D. Tacier, Solving underdefined systems of multivariate quadratic equations. Lect. Notes Comput. Sci. 2274, 211-227 (2002)

25. J.C. Lagarias, A.M. Odlyzko, Solving low-density subset sum problems. J. ACM 32, 229-246 (1985)

26. A.M. Youssef, Cryptanalysis of a knapsack-based probabilistic encryption scheme. Inf. Sci. 179(18), 3116-3121 (2009)

27. A.J. Menezes, P.C. Van Oorschot, S.A. Vanstone, Handbook of Applied Cryptography (CRC Press, Boca Raton, 1996)

28. E. Brickell, Solving low density knapsacks, in Proceedings of Crypto '83 (Plenum Press, New York, 1984), pp. 25-37

29. C.-P. Schnorr, H. H. Hörner, Attacking the Chor-Rivest cryptosystem by improved lattice reduction, in International Conference on the Theory and Applications of Cryptographic Techniques (Springer, New York, 1995), pp. 1-12

30. W. Bosma, J.J. Cannon, C. Playoust, The Magma Algebra System I: the user language. J. Symb. Comput. 24(3-4), 235-265 (1997) 